\title{
Diabetic Dyslipidemia - Role of Saroglitazar
}

\section{Anirban Majumder ${ }^{1}$ and Sanjay Chatterjee ${ }^{2 *}$}

${ }^{1}$ Associate Professor, Endocrinology unit, Department of Medicine, KPC Medical College, West Bengal University of Health Sciences, Kolkata, India ${ }^{2}$ Consultant Diabetologist, Apollo Gleneagles Hospital, Kolkata, India

\begin{abstract}
'Diabetic dyslipidemia' (combination of raised triglyceride levels, raised small-dense Low-density lipoprotein particles and low high-density lipoprotein cholesterol levels) is the most prominent risk factor of atherosclerosis and cardiovascular disease. Despite statin therapy and LDL-lowering, a high residual risk of cardiovascular events persists. High triglyceride levels and low high-density lipoprotein cholesterol levels singly and collectively increase the risk of cardiovascular events in type 2 diabetes mellitus and guidelines recommend modifying these secondary therapeutic targets to provide additional vascular protection.Addition of a fibrate or niacinareoptions for combination with a statin to reduce atherogenic dyslipidemia and are clearly effective at raising high-density lipoprotein cholesterol levels and lowering triglycerides.Addition of high dose omega-3 fatty acids (2-3 gm/day) with a statin are also effective in lowering triglyceride, but their effects on cardiovascular events remain uncertain and are complicated with safety issues. Saroglitazar is a novel dual Peroxisome Proliferator-Activated Receptors- $\alpha / y$ agonist and the first glitazar approved in the world for the treatment of diabetic dyslipidemia by Drug Controller General of India in June 2013. Saroglitazar (2 $\mathrm{mg}$ and $4 \mathrm{mg}$ ) therapy has shown significant $(45 \%)$ reduction in triglycerides, significant reduction of other atherogenic lipids (Low-density lipoprotein, very-low density lipoprotein, total cholesterol and apolipoprotein-B) and significant improvement of glycemic status, with relatively free from side effects. It has emerged with a hope to further reduce the incidence of cardiovascular disease among statin treated diabetic subjects. However, the trial populations were small and toxicity data may emerge with increasing use of this drug. Moreover, in absence of outcome studies and large multi-center longitudinal follow up data, the clinical cardiovascular efficacy is uncertain till date.
\end{abstract}

Keywords: Low-density lipoprotein; High-density lipoprotein; Triglyceride; Peroxisome proliferator-activated receptor

\section{Introduction}

Cardiovascular disease is the leading cause of premature morbidity and mortality worldwide [1]. The role of lipids in the causation of vascular disease is established in the past few decadesand pharmacotherapy with statins, targeting to lower the Low-density lipoprotein (LDL) cholesterol has proved a particularly effective intervention strategy [2,3]. However, in spite of statin therapy and LDL-lowering, a high residual risk of cardiovascular events persists [4], drawing attention to the need for additional effective preventive measures. Although several studies have shown associations of high-density lipoprotein (HDL) cholesterol and triglyceride (TG) concentrations with vascular risk, interventions that increase HDL cholesterol or reduce TG concentrations, or both, have not consistently shown benefit [5-7].

The potential role of peroxisome proliferator-activated receptor (PPAR) agonists to positively influence the cardiovascular disease risk in type 2 diabetes mellitus (T2DM) has remained an area of continuous medical interest and there is a strong need for a safe dual PPAR- $\alpha / \gamma$ agonist with effects in both lipids and glycemic parameters. Saroglitazar, is a dual PPAR- $\alpha / \gamma$ agonist, designed to optimize glycemic control and lipid parameters (reduces TG and increases HDL), and minimize PPAR-related adverse effects in the treatment of patients with T2DM [8]. In the following review we will focus on the role of Saroglitazar in dyslipidemia, particularly in T2DM. Saroglitazar (marketed by Zydus Cadila, Ahmedabad, India in the brand name Lipaglyn) is approved by Drug Controller General of India (DCGI) for treating diabetic dyslipidemia in June 2013 [9].

\section{Dyslipidemia and Cardiovascular Disease}

Dyslipidemia is one of the most prominent risk factor of atherosclerosis, leading to cardiovascular disease. Total cholesterol, non-HDL cholesterol, LDL cholesterol, apolipoprotein B, TG, HDL cholesterol, apolipoprotein A1, total cholesterol/HDL cholesterol, and apolipoprotein $\mathrm{B} /$ apolipoprotein $\mathrm{A} 1$ are all associated with increased risk of cardiovascular disease [10,11]. CVD is the major cause of morbidity with 2 - to 4 -fold increase in cardiovascular morbidity (coronary heart disease, stroke and peripheral vascular disease) in individuals with Type 2 Diabetes Mellitus (T2DM) compared to non-diabetic subjects and accounts for up to $80 \%$ of the mortality in T2DM [12]. The University Group Diabetes Program (UGDP) and the United Kingdom Prospective Diabetes Study (UKPDS), have found no relationship between glycemic control and diabetic macrovascular manifestations [13,14]. However, more recent clinical trials - the UKPDS 10 years follow-up study showed importance of glycemic control and its favorable influence on macrovascular outcome [15]. The STENO-2 study, a multifactorial intervention on cardiovascular risk factors, including an intensive control of lipids, glucose and blood pressure, reduces cardiovascular mortality [16]. Numerous studies consistently showed that pharmacological interventions that target the dyslipidemia and hypertension associated with T2DM, reduce risk of macrovascular complications in such patients $[16,17]$. Hence, we target conventional risk factors (e.g smoking, hypertension, dyslipidemia etc) to improve the CVD related morbidity and mortality in diabetic subjects.

Prevalence of dyslipidemia in Indian diabetic subjects are much higher compared to general population and is present in about 85.5$97.8 \%$ of the uncontrolled diabetic patients on enrolment and $60.6 \%$ of controlled well-treated diabetes [18]. Combined dyslipidemia with high TG and low HDL or high LDL and low HDL are the most common

*Corresponding authors: Sanjay Chatterjee, Consultant Diabetologist Apollo Gleneagles Hospital, Kolkata, India, Tel: 91-9831032310; E-mail: sanjay_doc@yahoo.co.in

Received August 27, 2014; Accepted September 24, 2014; Published September 26, 2014

Citation: Majumder A, Chatterjee S (2014) Diabetic Dyslipidemia - Role of Saroglitazar. Med chem 4: 684-687. doi:10.4172/2161-0444.1000212

Copyright: (c) 2014 Majumder A, et al. This is an open-access article distributed under the terms of the Creative Commons Attribution License, which permits unrestricted use, distribution, and reproduction in any medium, provided the original author and source are credited. 
pattern of dyslipidemia and isolated low HDL level or isolated high TG are the second most common pattern of dyslipidemia among Indian diabetics [19].

\section{Beyond LDL-C: The non-HDL-C and TG}

Aggressive LDL-cholesterol lowering treatment has become the mainstay of lipid-lowering strategies for the last 2 decades.Despite achieving target LDL-cholesterol, statins reduce CVD risk by $25-35 \%$. Despite the best evidence-based strategies, including high-dose statin therapy, it is clear that there persists an unacceptably high residual risk of cardiovascular events, especially in T2DM [20]. High TG and low HDL cholesterol levels have been shown to singly and collectively boost the risk of cardiovascular events independent of conventional risk factors [21]. Furthermore, guidelines recommend modifying high TG and low HDL as secondary therapeutic targets to provide additional vascular protection [14].

Given the high residual risk of cardiovascular events beyond effective LDL-lowering, identification of additional lipid biomarkers (HDL \& TG) independently linked to vascular events, and the very limited effect of statins on modifying these lipid markers (HDL \& TG), there is mounting interest in optimizing these additional lipid biomarkers via additional therapies. Addition of a fibrate, niacin or omega-3 fatty acids are all options for combination with a statin to further reduce atherogenic dyslipidemia. Several emerging treatments, the next generation peroxisome proliferator-activated receptor alpha agonists, cholesteryl ester transfer protein inhibitors and monoclonal antibody therapy targeting proprotein-convert ase subtilisin/kexin type 9 may offer promise [4]. However, long-term outcomes and safety data are clearly needed.

\section{Limitation of Current Pharmaco-Therapeutic Strategies}

Multiple lipid lowering medications have been developed that effectively reduce TG with expectation to reduce the morbidity and mortality from CVD.

\section{Fibrates}

Fenofibrate (peroxisome proliferator-activated receptor- $\alpha$ [PPAR $\alpha]$ agonists) are routinely used to reduce TG. In the FIELD (Fenofibrate Intervention and Event Lowering in Diabetes) study, TG lowering therapy with fenofibrate did not significantly reduce the overall risk of the primary outcome of coronary events [22]. In a systematic metaanalysis of 18 trials with a total 45,058 participants, the fibrates was found to reduce the risk of major cardiovascular events [23]. However, the magnitude of the effect is moderate and clinically meaningful reductions in risk was achieved only in high-risk individuals and speciallyin those with combined dyslipidemia. However, the efficacy of the current fibrates may be limited by uncertainties relating to the optimal level of PPARa-agonism, agonist-specific biologic responses, the side effects of current synthetic agonists and of course the relevance for patients already on statin therapy, specially in view of increased risk of muscle-related adverse events.

\section{Niacin}

TheExtended-release(ER) niacin (1.5-2 g/day) showed the positive impact on HDL and TG values but did not demonstrate a beneficial effect on the primary endpoints (coronary heart disease death, nonfatal myocardial infarction, ischemic stroke, hospitalization for acute coronary syndrome, or symptom-driven coronary or cerebral revascularization) in patients with CVD and optimally treated with a statin but with residual atherogenic dyslipidaemia in the AIM-HIGH (Atherothrombosis Intervention in Metabolic Syndrome With Low
HDL/High Triglycerides: Impact on Global Health Outcomes) trial [24]. The much larger HPS2-THRIVE (Heart Protection Study 2-Treatment of HDL to Reduce the Incidence of Vascular Events) also failed to show a benefit on clinical outcomes with a different niacin formulation (combined with laropiprant) [25]. Moreover, treatment with niacin did adversely affect patients with higher risk of gastrointestinal events, musculoskeletal events, infection, skin-related adverse events, bleeding and new onset diabetes, leading to subsequent world-wide withdrawal of this therapy [26].

\section{Omega-3 fatty acids}

JELIS trial (Japan Eicosapentaenoic acid Lipid Intervention Study) showed a $19 \%$ risk reduction in major coronary events [27]. However, Alpha-Omega trial (n-3 Fatty Acids and Cardiovascular Events after Myocardial Infarction) failed to show a benefits with omega-3 fatty acids. "ORIGIN trial (Outcome Reduction With Initial Glargine Intervention)" that included patients with dysglycemia (N: 12,536) showed no cardiovascular benefits in the study population exposed to omega 3 fatty acid compared to placebo during the study period of over 6 years [28]. Moreover, there isevidence from recent trials to suggest a possible link between omega- 3 fatty acids and increased risk of prostate cancer $[29,30]$.

\section{Cholesteryl ester transfer protein inhibitors (CETP inhibitors)}

Strategies to increase HDL through inhibition of CETP have prematurely terminated because of an increased risk of death and cardiac events in patients receiving torcetrapib in the ILLUMINATE study [31]. Dalcetrapib, another CETP inhibitor, did not reduce the risk of recurrent cardiovascular events in patients who had a recent acute coronary syndrome, in OUTCOMES study [32].

\section{Saroglitazar Therapy}

There are gaps in the treatment of high TG with each of these agents mentioned above in respect of the effectiveness of the therapy in reducing CVD and concern about safety. Saroglitazar is a dual Peroxisome Proliferator-Activated Receptors- $\alpha / \gamma$ agonist, the first glitazar approved in the world and has emerged with a new hope to effectively reducing diabetic dyslipidemia with relative free of side effects specially with no increase of body weight [8].

Peroxisome Proliferator-Activated Receptors (PPARs) are nuclear lipid-activated transcription factors that regulate the expression of genes involved in the control of lipid and lipoprotein metabolism, glucose homeostasis and inflammatory processes. There are three PPAR isoforms with different pharmacological activities:PPAR $\alpha$ plays a key role in lipid metabolism, whereas PPAR $\gamma$ and PPAR $\delta$ are critical players in regulating energy metabolism in adipose tissue and muscle and hence consideredfor the treatment of insulin resistance. So dual PPAR agonists with selective activity for PPAR $\alpha / \gamma$ or $\mathrm{PPAR} \alpha / \delta$, may offer an opportunity to manage several areas of dysmetabolism [33].

Saroglitazar is currently the only agent of this class approved for the treatment of diabetic dyslipidemia in India (by Drug Controller General of India in June 2013) and the approval was based on the results obtained from clinical studies, which were conducted for more than eight years. Saroglitazar is a novel PPAR agonist with predominant PPARa and moderate PPAR $\gamma$ agonist activity. It is a non-thiazolidinedione and non-fibrate molecule and belongs to aryl alkoxy propionic acid class. Saroglitazar is well absorbed and has predictable pharmacokinetic parameters [34]. The elimination half-life of Saroglitazar is long and is supportive of once daily dosing. After oral administration, saroglitazar does not appear in urine samples, indicating that saroglitazar is not eliminated via the renal route and is mainly eliminated by the 
enterohepatic route [34]. Saroglitazar is well tolerated up to a dose of $128 \mathrm{mg}$ in the single-ascending dose study and this highest tested dose of $128 \mathrm{mg}$ provide a safety margin of over 32 -fold as the therapeutic dose is $2-4 \mathrm{mg}$ per day [34]. Pharmacokinetics, safety and tolerability of Saroglitazar on a very limited number of healthy volunteers $(n=5$ or 6 , as available) showed no serious adverse events and no clinically relevant trends or changes in laboratory values or ECG over time [34]. Only two serious adverse events were reported with $4 \mathrm{mg}$ saroglitazar unrelated with the study drug. Pyrexia, dyspepsia and gastritis were the most common adverse events reported and were mild to moderate in intensity [35]. There were no significant changes in hemoglobin, liver enzymes, renal function, CPK, and high-sensitivity C-reactive protein with saroglitazar therapy for 12 weeks of treatment $[34,35]$.

Saroglitazar ( $2 \mathrm{mg}$ and $4 \mathrm{mg}$ ) therapy has shown $45 \%$ reduction in triglycerides and a significant reduction of other atherogenic lipids (LDL-C, VLDL-C, TC, Apo B) [8,35]. Saroglitazar showed a doserelated decrease in fasting glucose (mean $11.3 \mathrm{mg} / \mathrm{dl}$ reduction in $2 \mathrm{mg}$ arm and mean $22.6 \mathrm{mg} / \mathrm{dl}$ reduction in $4 \mathrm{mg}$ arm) and HbAlc (mean $0.3 \%$ reduction in both $2 \mathrm{mg}$ and $4 \mathrm{mg}$ arm), very similar to Pioglitazone $45 \mathrm{mg}$ therapy in a 24 week trial without any significant changes from baseline in any of the safety laboratory findings and importantly without significant change in bodyweights compared to pioglitazone (mean increase of body weight $1.6 \mathrm{~kg}$ ) arm. The decrease in $\mathrm{HbAlclevel}$ was comparable to pioglitazone $(45 \mathrm{mg})$ and the reduction depended on the baseline value withhigher the value, the greater the decrease [8]. Saroglitazar were evaluated in diabetic dyslipidemic patients insufficiently controlled with statin therapy and showed pronounced beneficial effect on both the lipid (significantly reduced mean plasma triglyceride levels by $-45.5 \pm 3.03 \%$ and $-46.7 \pm 3.02 \%$, with 2 -mg and 4-mg dose respectively, along with significant decrease in levels of nonHDL cholesterol, VLDL, total cholesterol)and glycaemic parameters [35]. In summary, Saroglitazar is the first drug having both lipid and glucose lowering effects ensuring a comprehensive management of diabetes and dyslipidemia.

\section{Limitation of Saroglitazar}

Saroglitazar is indicated as adjunctive therapy to diet and statin for treatment of adult diabetic patients with hypertriglyceridemia. Markedly elevated levels of TG (e.g. $>500 \mathrm{mg} / \mathrm{dL}$ ) even after glycemic control may increase the risk of developing pancreatitis. The effect of Saroglitazar therapy on reducing this risk has not been adequately studied.

The role of Glitazars in atherogenic dyslipidemia and diabetes has remained an area of interest for last two decades and appears suitable for diabetic patients on statin therapy who have residual cardiovascular risk.The development of most of these glitazars were complicated with various side effects and was dropped, Faglitazar, due to significant edema [36], Ragaglitazar due to its carcinogenic potential in rodent models [37], Muraglitazar due to significant cardiovascular side effects [38] and Tesaglitazar due to its bone marrow \& renal toxicities [39]. Although these tested molecules resulted in adverse events, these have been compound specific and of diverse origin, rising hopes for Saroglitazar, that could mitigate these side effects and yet have a positive influence on insulin sensitivity and dyslipidaemia.

Diabetic dyslipidemia are targeted for the specific reason to reduce the cardiovascular morbidity and mortality. Though Saroglitazar was shown to improve diabetic dyslipidemia butwas not shown to reduce coronary heart disease in a large, randomized controlled trial of patients with T2DM.

\section{Conclusion}

Despite best evidence-based strategies and achievement of treatment goals for LDL cholesterol, blood pressure, and glycemia according to current standards of care, it is clear that there persists an unacceptably high residual risk of CVD events, especially in T2DM.It is advisable to stick to statins alone but residual risk should be addressed after optimization of LDL cholesterol. TG and HDL concentrations repeatedly shown to significantly predict future risk for cardiovascular disease and obviously considered important as therapeutic targets. However, pharmacological interventional trials with Fibrates or Niacin or Omega-3 fatty acids or CETP inhibitors, targeting TG and HDL repeatedly failed to show a benefit on CVD events and were complicated with safety issues leading to premature termination of many trials.

Saroglitazar is an approved therapy for diabetic dyslipidemia in India and it improves not only TG and HDL concentrations but also glycemic parameters. Although this review highlights clinical and experimental data that provide evidence for favorable effects of Saroglitazar on diabetic dyslipidemia, the lack of outcome studies limit our knowledge about their clinical cardiovascular efficacy and toxicity data may emerge with increasing use. Large multi-center longitudinal studies are required to demonstrate the safety \& cardiovascular benefits of Saroglitazar and will clarify whether this dual PPAR agonist live up to their promise as vasculo-protective agent.

\section{References}

1. Neal B, Chapman N, Patel A (2002) Managing the global burden of cardiovascular disease. Eur Heart J Supplements 4 (Suppl F): F2-F6.

2. Sever PS, Dahlof B, Poulter NR, Wedel H, Beevers G, et al. (2003) Prevention of coronary and stroke events with atorvastatin in hypertensive patients who have average or lower-than-average cholesterol concentrations, in the AngloScandinavian Cardiac Outcomes Trial-Lipid Lowering Arm (ASCOT-LLA): a multi centre randomised controlled trial. Lancet 361: 1149-1158.

3. Randomised trial of cholesterol lowering in 4444 patients with coronary heart disease: the Scandinavian Simvastatin Survival Study (4S)(1994) Lancet 344: 1383-1389.

4. Fruchart JC, Sacks FM, Hermans MP, Assmann G, Brown WV, et al. (2008) The Residual Risk Reduction Initiative: a call to action to reduce residual vascular risk in dyslipidaemic patient. DiabVasc Dis Res 5: 319-335.

5. Guyton JR, Slee AE, Anderson T, Fleg JL, Goldberg RB, et al. (2013) Relationship of lipoproteins to cardiovascular events: theAIM-HIGH Tria (Atherothrombosis Intervention in Metabolic SyndromeWith Low HDL/High Triglycerides and Impact on Global Health Outcomes). J Am CollCardiol 62: 1580-1584.

6. HPS2-THRIVE Collaborative Group (2013) HPS2-THRIVE randomized placebo-controlled trial in 25673 high-risk patients of ER niacin/laropiprant: trial design, pre-specified muscle and liver outcomes, and reasons for stopping study treatment. Eur Heart J 34: 1279-1291.

7. Scott R, O'Brien R, Fulcher G, Pardy C, d'Emden M, et al. (2009) Effects of Fenofibrate Treatment on Cardiovascular Disease Risk in 9,795 Individuals With Type 2 Diabetes and Various Components of the Metabolic Syndrome: the Fenofibrate Intervention and Event Lowering in Diabetes (FIELD) study. Diabetes Care 32: 493-498.

8. Pai V, Paneerselvam A, Mukhopadhyay S, Bhansali A, Kamath D, et al. (2014) A Multicenter, Prospective, Randomized, Double-blind Study to Evaluate the Safety and Efficacy of Saroglitazar 2 and $4 \mathrm{mg}$ Compared to Pioglitazone 45 $\mathrm{mg}$ in Diabetic Dyslipidemia (PRESS V). J Diabetes Sci Technol 8: 132-141.

9. Agrawal R (2014) The first approved agent in the Glitazar's Class: Saroglitazar. Curr Drug Targets 15: 151-155.

10. Nordestgaard BG,Langsted A, Freiberg JJ (2009) Nonfasting hyperlipidemia and cardiovascular disease. Curr Drug Targets 10: 328-335.

11. Stalenhoef AF, de Graaf $J$ (2008) Association of fasting and nonfasting serum triglycerides with cardiovascular disease and the role of remnant-like lipoproteins and small dense LDL. Curr Opin Lipidol 19: 355-361. 
12. Blendea MC, McFarlane SI, Isenovic ER, Gick G, Sowers JR (2003) Heart disease in diabetic patients. Curr Diab Rep 3: 223-229.

13. Cornfield J (1971) The University Group Diabetes Program. A further statistical analysis of the mortality findings. JAMA 217: 1676-1687.

14. King P, Peacock I, Donnelly R (1999) The UK prospective diabetes study (UKPDS): clinical and therapeutic implications for type 2 diabetes. $\mathrm{Br} \mathrm{J}$ Clin Pharmacol 48: 643-648.

15. Holman RR, Paul SK, Bethel MA, Matthews DR, Neil HA (2008) 10-year followup of intensive glucose control in type 2 diabetes. N Engl J Med 359: 1577 1589.

16. Gaede P, Lund-Andersen H, Parving HH, Pedersen O (2008) Effect of a multifactorial intervention on mortality in type 2 diabetes. $\mathrm{N}$ Engl $\mathrm{J}$ Med 358: 580-591.

17. Beckman JA,Creager MA, Libby $P$ (2002) Diabetes and atherosclerosis: epidemiology, pathophysiology, and management. JAMA 287: 2570-2581.

18. Pandya H, Lakhani JD, Dadhania J, Trivedi A (2012) The Prevalence and Pattern of Dyslipidemia among Type 2 Diabetic Patients at Rural Based Hospital in Gujarat, India. Indian J Clin Pra 22: 36-44.

19. Parikh RM, Joshi SR, Menon PS, Shah NS (2010) Prevalence and pattern of diabetic dyslipidemia in Indian type 2 diabetic patients: Diabetes \& Metabolic Syndrome. Clinical Research \& Reviews 4:10-12.

20. Fruchart JC,Davignon J, Hermans MP, Al-Rubeaan K, Amarenco P, et al. (2014) Residual macrovascular risk in 2013: what have we learned? CardiovascDiabetol 13: 26.

21. Barter P,Gotto AM, LaRosa JC, Maroni J, Szarek M, et al. (2007) HDL cholesterol, very low levels of LDL cholesterol, and cardiovascular events. N Engl J Med 357: 1301-1310.

22. Keech A, Simes RJ, Barter P, Best J, Scott R, et al. (2005) Effects of longterm fenofibrate therapy on cardiovascular events in 9795 people with type 2 diabetes mellitus (the FIELD study): Randomized controlled trial. Lancet 366:1849-1861.

23. Jun M, Foote C, Lv J, Neal B, Patel A, et al. (2010) Effects of fibrates on cardiovascular outcomes: a systematic review and meta-analysis. Lancet 375 : 1875-1884.

24. AIM-HIGH Investigators, Boden WE, Probstfield JL, Anderson T, Chaitman $\mathrm{BR}$, et al. (2011) Niacin in patients with low HDL cholesterol levels receiving intensive statin therapy. N Engl J Med 365: 2255-2267.

25. HPS2-THRIVE Collaborative Group, Landray MJ, Haynes R, Hopewell JC, Parish S, et al. (2014) Effects of extended-release niacin with laropiprant in high-risk patients. N Engl J Med 371: 203-212.

26. Merck Provides Update on Next Steps for TREDAPTIVE TM (extended-release niacin/ laropiprant).

27. Yokoyama M,Origasa H, Matsuzaki M, Matsuzawa Y, Saito Y, et al. (2007) Effects of eicosapentaenoic acid on major coronary events in hypercholesterolaemic patients (JELIS): a randomised open-label, blinded endpoint analysis. Lancet 369: 1090-1098.

28. ORIGIN Trial Investigators, Bosch J, Gerstein HC, Dagenais GR, DÃaz R, et al. (2012) n-3 fatty acids and cardiovascular outcomes in patients with dysglycemia. N Engl J Med 367: 309-318.

29. Brasky TM,Darke AK, Song X, Tangen CM, Goodman PJ, et al. (2013) Plasma phospholipid fatty acids and prostate cancer risk in the SELECT trial. J Natl Cancer Inst 105: 1132-1141.

30. Brasky TM, Till C, White E, Neuhouser ML, Song X, et al. (2011) Serum phospholipid fatty acids and prostate cancer risk: results from the prostate cancer prevention trial. Am J Epidemiol 173: 1429-1439.

31. Barter PJ, Caulfield M, Eriksson M, Grundy SM, Kastelein JJ, et al. (2007) Effects of torcetrapib in patients at high risk for coronary events. N Engl J Med 357: 2109-2122.

32. Schwartz GG, Olsson AG, Abt M, Ballantyne CM, Barter PJ, et al. (2012) Effects of dalcetrapib in patients with a recent acute coronary syndrome. N Engl J Med 367: 2089-2099.

33. Fruchart JC (2013) Selective peroxisome proliferator-activated receptora modulators (SPPARMa): the next generation of peroxisome proliferatoractivated receptor a-agonists. Cardiovasc Diabetol 12: 82.

34. Jani RH,Kansagra K, Jain MR, Patel H (2013) Pharmacokinetics, safety, and tolerability of saroglitazar (ZYH1), a predominantly PPAR $\alpha$ agonist with moderate PPARy agonist activity in healthy human subjects. Clin Drug Investig 33: 809-816.

35. Jani RH, Pai V, Jha P, Jariwala G, Mukhopadhyay S, et al. (2014) A multicenter prospective, randomized, double-blind study to evaluate the safety and efficacy of Saroglitazar 2 and $4 \mathrm{mg}$ compared with placebo in type 2 diabetes mellitus patients having hypertriglyceridemia not controlled with atorvastatin therapy (PRESS VI). Diabetes Technol Ther 16: 63-71.

36. Henke BR, Blanchard SG, Brackeen MF, Brown KK, Cobb JE, et al. (1998) $\mathrm{N}$-(2-Benzoylphenyl)-L-tyrosine PPARgamma agonists. 1. Discovery of a nove series of potent antihyperglycemic and antihyperlipidemic agents. J Med Chem 41: 5020-5036.

37. Egerod FL, Nielsen HS, Iversen L, Thorup I, Storgaard T, et al. (2005) Biomarkers for early effects of carcinogenic dual-acting PPAR agonists in rat urinary bladder urothelium in vivo. Biomarkers 10: 295-309.

38. Kendall DM, Rubin CJ, Mohideen P, Ledeine JM, Belderet R, et al. (2006) Improvement in glycemic control, triglycerides, and HDL cholesterol levels with muraglitazar, a dual (alpha/gamma) peroxisome proliferator-activated receptor activator, in patients with type 2 diabetes inadequately controlled with metformin monotherapy: A double blind, randomized, pioglitazone-comparative study. Diabetes Care 29: 1016-1023.

39. Fagerberg B, Edwards S, Halmos T, Lopatynsky J, Schuster H, et al. (2005) Tesaglitazar, a novel dual peroxisome proliferator-activated receptor alpha/ gamma agonist, dose-dependently improves the metabolic abnormalities associated with insulin resistance in a non-diabetic population. Diabetologia 48: 1716-1725. 